\title{
Third molar agenesis in modern humans with and without agenesis of other teeth
}

\author{
Maya Scheiwiller ${ }^{1}$, Elias S Oeschger ${ }^{1}$, Nikolaos Gkantidis ${ }^{\text {Corresp. } 1}$ \\ ${ }^{1}$ Department of Orthodontics and Dentofacial Orthopedics, University of Bern, Bern, Switzerland \\ Corresponding Author: Nikolaos Gkantidis \\ Email address: nikosgant@yahoo.gr
}

Background. The number of teeth in the human dentition is of interest both from developmental and evolutionary aspects. The present case-control study focused on the formation of third molars in modern humans aiming to shed more light on the most variable tooth class in the dentition.

Materials and Methods. For this reason, we investigated third molar formation in a sample of 303 individuals with agenesis of teeth other than third molars (agenesis group) and compared it to a sex and age matched control group of 303 individuals without agenesis of teeth other than third molars.

Results. The prevalence of third molar agenesis in the agenesis group was $50.8 \%$, which is significantly higher than the $20.5 \%$ in the control group ( $p<0.001$ ). The chance of a missing third molar in the agenesis group was increased by 38.3\% ( $p<0.001)$, after controlling for the agenesis in other teeth factor. When considering the amount of missing third molars per individual, a clear tendency towards more missing third molars was evident in the agenesis group compared to the control group. The frequency of bilaterally missing third molars in the agenesis group was $29 \%$ in the maxilla, as well as in the mandible, which is about three times higher than the frequency of unilaterally missing third molars ( $p$ $<0.001$ ). In the control group, bilaterally missing third molars occurred in $8.6 \%$ in the maxilla and $8.9 \%$ in the mandible.

Conclusion. The present results indicate that genetic factors involved in tooth agenesis affect also the dentition as a whole. Furthermore, the third molars are more vulnerable to factors involved in agenesis of other teeth and they are more often affected as a whole. These findings seem to be associated with the evolutionary trend in humans towards reduced molar number. 
1 Third molar agenesis in modern humans with and without agenesis of other

2 teeth

3 Maya Scheiwiller ${ }^{1}$, Elias S. Oeschger ${ }^{1}$, Nikolaos Gkantidis ${ }^{1}$

4

5 'Department of Orthodontics and Dentofacial Orthopedics, University of Bern, Freiburgstrasse 7, 6 CH-3010, Bern

7

8 Corresponding Author

9 Nikolaos Gkantidis, Department of Orthodontics and Dentofacial Orthopedics, University of

10 Bern, CH-3010, Freiburgstrasse 7, Bern, Switzerland

11 Tel.: +41 (0) 3163225 92, Fax: +41 (0) 316329869

12 Email: nikolaos.gkantidis@zmk.unibe.ch; nikosgant@yahoo.gr 


\section{Abstract}

Background. The number of teeth in the human dentition is of interest both from developmental and evolutionary aspects. The present case-control study focused on the formation of third molars in modern humans aiming to shed more light on the most variable tooth class in the dentition.

Materials and Methods. For this reason, we investigated third molar formation in a sample of 303 individuals with agenesis of teeth other than third molars (agenesis group) and compared it to a sex and age matched control group of 303 individuals without agenesis of teeth other than third molars.

Results. The prevalence of third molar agenesis in the agenesis group was $50.8 \%$, which is significantly higher than the $20.5 \%$ in the control group $(\mathrm{p}<0.001)$. The chance of a missing third molar in the agenesis group was increased by $38.3 \%(\mathrm{p}<0.001)$, after controlling for the agenesis in other teeth factor. When considering the amount of missing third molars per individual, a clear tendency towards more missing third molars was evident in the agenesis group compared to the control group. The frequency of bilaterally missing third molars in the agenesis group was $29 \%$ in the maxilla, as well as in the mandible, which is about three times higher than the frequency of unilaterally missing third molars $(p<0.001)$. In the control group, bilaterally missing third molars occurred in $8.6 \%$ in the maxilla and $8.9 \%$ in the mandible.

Conclusion. The present results indicate that genetic factors involved in tooth agenesis affect also the dentition as a whole. Furthermore, the third molars are more vulnerable to factors involved in agenesis of other teeth and they are more often affected as a whole. These findings seem to be associated with the evolutionary trend in humans towards reduced molar number. 


\section{INTRODUCTION}

Tooth agenesis is the congenital absence of one or more teeth. In the primary dentition, the prevalence ranges between $0.1 \%$ and $0.2 \%$. However, in the permanent dentition tooth agenesis is prevalent in $6.4 \%$ of the overall population, with similar occurrence in the two jaws (Khalaf et al. 2014). There is a large variation between different population groups and studies (Khalaf et al. 2014).

Tooth agenesis studies generally exclude third molars, due to the high frequency of their absence (Khalaf et al. 2014). Agenesis of third molars is more or less considered a physiologic finding or an evolutionary adaptation of the dentition rather than a developmental disturbance (Koussoulakou et al. 2009). The third molar is the last tooth to develop in the dentition and is characterized by the variability in time of formation and by its diversity in presence or absence (Banks 1934; Celikoglu et al. 2010). The worldwide average of third molar agenesis is $22.6 \%$, with Asian populations showing the highest rate of 29.7\% (Carter \& Worthington 2015).

A wide range of studies shows that the agenesis of third molars correlates with the number of other teeth in the dentition. According to Garn et al. (Garn et al. 1962), the chance of another tooth to be missing is raised thirteen-fold if at least one-third molar is missing. More recent studies point in the same direction, though with much reduced effect sizes (Bredy et al. 1991; Celikoglu et al. 2011; Endo et al. 2015). Endo et al. (Endo et al. 2013) reported a significant association between missing third molars and bilateral agenesis of other teeth. Other researchers focused on the agenesis of specific teeth and third molar agenesis (Abe et al. 2010; Garib et al. 2010; Garib et al. 2009).

So far, various studies have investigated the association between missing third molars and agenesis of other teeth, but on limited tooth agenesis samples. Furthermore, most relevant studies tested Asian populations. Thus, we performed a study in a large sample of European subjects, aiming to investigate third molar formation in individuals with and without agenesis of other teeth. To obtain a robust sample, we selected a large number of individuals with agenesis of teeth other than third molars and compared it to a matched group without agenesis of teeth other than third molars. The current approach offers the opportunity to assess previously tested, but also novel questions, relevant to the study hypothesis, with adequate sample sizes. The primary null hypothesis was that there is no difference in third molar agenesis patterns between individuals who have agenesis in teeth other than third molars, and those who do not.

\section{Materials ANd Methods}

In this case-control study, we followed the STROBE guidelines for reporting observational studies (von Elm et al. 2008).

The ethical approval was provided by the Ethics Commission of the Canton of Bern, Switzerland (Project-ID: 2018-01340) and the Research Committee of the School of Dentistry, National and 
72 Kapodistrian University of Athens, Greece (Project-ID: 281, 2/9/2016). The need for informed

73 consent was waived for part of the sample and was obtained for the rest.

74 Study sample

75 Consecutive orthodontic records of various time periods within a 12-year period (2006 - 2018,

76 depending on the place of sample collection) were searched for eligible subjects at the following

77 clinics: A) University of Bern, Switzerland b) University of Athens, Greece, c) two private

78 practices in Athens and two in Thessaloniki, Greece, and d) one private practice in Biel,

79 Switzerland. Sample collection was performed at the place of data generation by colleagues who

80 were blinded to the aim of this study.

81 The sample was collected based on the following inclusion criteria:

82 - Individuals with an age between 12.5 and 40 years

83 - Individuals with and without agenesis of teeth other than third molars for the agenesis and the

84 control group, respectively

- European ancestry

- No syndromes, systemic diseases or other defects that affect the craniofacial complex development, as reported in the subjects' medical records

- Adequate quality panoramic radiographs for identification of missing teeth (Figure S1)

- No individuals where the cause of missing teeth was unclear

- No individuals where the presence or absence of teeth could not be confirmed

The minimum age limit of 12.5 years was determined according to previous studies that evaluated the correlation between chronological age and the degree of third molar mineralization (Caldas et al. 2011; de Oliveira et al. 2012; Karataş et al. 2013; Soares et al. 2015; Zandi et al. 2015). They showed that in $95 \%$ of cases, Demirjian's stage A was observed at the age of 12.5 or younger, which means that the mineralization of third molar crowns has already started and is clearly visible on the panoramic radiographs.

Finally, the panoramic radiographs of 303 individuals with agenesis of teeth other than third molars (agenesis group) were selected from a large orthodontic sample of approximately 10.000 individuals, based on availability. A control group of 303 individuals without agenesis of teeth other than third molars, matched for age (within 6 months) and sex was formed from the same archives. All other inclusion criteria for the control group, were the same as mentioned above for the agenesis group.

\section{Data extraction}

After reviewing the orthodontic files (medical and dental history, intraoral and extraoral photos, radiographs) at the place of sample collection, the relevant data were recorded in an Excel sheet (Microsoft Excel, Microsoft Corporation, Redmond WA, USA) in a standardized manner. To 
107 identify tooth agenesis, the panoramic radiographs were digitized and viewed on screen. A single

108

109

110

111

112

113

114

115

116

117

118

119

120

121

122

123

124

125

126

127

128

129

130

131

132

133

134

135

136

137

138

139

140

141

142

researcher (M.S.) performed the data extraction procedure of the entire sample in terms of missing teeth, and repeated it for 40 randomly selected subjects (https://www.random.org/) following a 1-month washout period. In case of disagreement, the radiographs were controlled by all authors and a consensus was reached.

To record tooth agenesis patterns, the TAC (Tooth Agenesis Code) system was used (van Wijk $\&$ Tan 2006). This system assigns a binary value to each tooth providing a unique numeric value for each pattern. Each dental quadrant is analyzed separately, and thus, the combined values assigned to each of the quadrants (q1, q2, q3, and q4) represent a unique tooth agenesis pattern (van Wijk \& Tan 2006).

\section{Statistical analysis}

All statistical analyses were conducted with SPSS software (IBM SPSS Statistics for Windows, Version 23.0. Armonk, NY: IBM Corp). Descriptive statistics were also calculated through the Tooth Agenesis Code Data Analysis Tool (http://www.toothagenesiscode.com/, last accessed 15 May 2019). Intra-rater agreement was evaluated through the percentage of different patterns identified in the two repeated assessments. The two-tailed Pearson's Chi square test was used to assess differences in the frequencies observed in the control and the agenesis samples. The Spearman's correlation coefficient was used to investigate the relation of the number of agenesis of teeth other than third molars to the number of third molar agenesis, overall, as well as within quadrants.

\section{Results}

\section{Method error}

The Intra-rater agreement between repeated tooth agenesis pattern identification was $97.5 \%$.

\section{Agenesis group without considering third molars}

In the 303 individuals (170 females, 133 males) of the agenesis sample, in total 799 teeth, other than third molars, were congenitally missing. In $38.6 \%$ of the sample one tooth, in $33.3 \%$ two, and in $7.9 \%$ three teeth were missing (Table S1). The incidence for missing teeth in the maxilla was $57.1 \%$, compared to $68.6 \%$ in the mandible $(\mathrm{p}=0.079)$. The most common missing tooth was the mandibular second premolar $(29.3 \%)$, followed by the maxillary lateral incisor $(21.0 \%)$, and the maxillary second premolar (14.0\%; Table 1$)$.

Regarding tooth symmetry, the teeth that were most often symmetrically missing in the maxilla were the lateral incisors $(19.5 \%)$ followed by the second premolars $(14.2 \%)$. In the mandible, the second premolars were missing bilaterally in $25.4 \%$, followed by the central incisors in $7.9 \%$ (Table 2).

The most common agenesis patterns in the maxilla were bilaterally missing lateral incisors $(23.1 \%)$, followed by bilaterally missing second premolars $(12.7 \%)$. In the mandible, the most

PeerJ reviewing PDF | (2020:06:50473:2:0:NEW 15 Oct 2020) 
143

144

145

146

147

148

149

150

151

152

153

154

155

156

157

158

159

160

161

162

163

164

165

166

167

168

169

170

171

172

173

174

175

176

177

178

179

common patterns were bilateral agenesis of second premolars in $27.9 \%$, followed by unilateral agenesis of the right second premolar (17.3\%). In the whole dentition, bilateral agenesis of maxillary lateral incisors occurred most often $(11.2 \%)$, followed by bilateral agenesis of mandibular second premolars $(10.2 \%$; Table 3$)$.

\section{Third molar agenesis in the agenesis and the control group}

The prevalence of third molar agenesis in the agenesis group was $50.8 \%$, which is significantly larger than the $20.5 \%$ in the control group $(\mathrm{p}<0.001) .418$ third molars were congenitally missing in the agenesis group $(n=303)$ compared to 144 in the control group $(n=303)$. If the probability of third molar agenesis in the agenesis group was equal to that of teeth other than third molars, this would increase the value of 144 missing third molars, observed in the control group, by 114. Consequently, 258 missing third molars would have been expected in the agenesis group. This value is significantly lower than the actual value observed $(418 ; \mathrm{p}<0.001)$. Thus, the chance of a missing third molar in the agenesis group is increased by $38.3 \%$, compared to controls.

In the agenesis group, there was a significant, though weak correlation, of the total number of other missing teeth to the total number of missing third molars (rho $=0.31, p<0.001)$. Similarly, very weak correlations were identified when third molar agenesis was correlated to the number of other tooth agenesis within quadrants $(\mathrm{Q} 1$ : $r h o=0.16, \mathrm{p}=0.006 ; \mathrm{Q} 2$ : rho $=0.14, \mathrm{p}=0.015$; $\mathrm{Q} 3$ : rho $=0.20, \mathrm{p}=0.001 ; \mathrm{Q} 4: \mathrm{rho}=0.29, \mathrm{p}=0.001)$.

The frequency of bilaterally missing third molars in the agenesis group was $29 \%$ in the maxilla, as well as in the mandible. This is about three times higher than the frequency of unilaterally missing third molars (maxilla: $9.9 \%, \mathrm{p}<0.001$, mandible: $11.9 \%, \mathrm{p}<0.001$; Table 2 ). The ratio of bilateral to unilateral third molar agenesis was significantly higher in the agenesis group compared to the control group (maxilla: 2.93 vs. 1.53 , respectively, $\mathrm{p}<0.001$; mandible: 2.44 vs. 1.29, respectively, $\mathrm{p}<0.001$; Table 2).

In the tooth agenesis group, symmetrical third molar agenesis occurred in a similar manner within jaws (29\% within each jaw), between jaws (right side: $24 \%$, left side: $24 \%$ ), or crossed quadrant (q1 vs. q3: 22.1\%; q2 vs q4: 24.4\%) ( $p>0.05$; Table 4). The same was true for the control groups ( $\mathrm{p}>0.05$; Table 4$)$, though the prevalence of all respective symmetrical patterns was much lower (range: $6.6-8.9 \%, \mathrm{p}<0.001$ ).

In both groups, there was no statistically significant difference between the number of missing third molars in the different quadrants (Chi square test, $\mathrm{p}>0.05$; Table 1). The agenesis group differed significantly from the control group in the distribution of the number of missing third molars $(\mathrm{p}<0.001)$. There is a clear tendency towards more missing third molars in the agenesis group compared to the controls. The agenesis group has 1.55, 2.14, 3.80, and 3.48 times higher possibility of having one, two, three, or four missing third molars respectively, when compared to the control group (Figure 1). 
180 Table S2 shows the most common patterns of tooth agenesis in the agenesis group, including

181

182

183

184

185

186

187

188

189

190

191

192

193

194

195

196

197

198

199

200

201

202

203

204

205

206

207

208

209

210

211

212

213

214

215

216

217

third molars. In the maxilla, the lateral incisors were most commonly missing in $14.1 \%$, followed by bilaterally missing third molars in $13.6 \%$ of the sample. In the mandible, the second premolars were most commonly missing bilaterally in $12.8 \%$ of the sample, followed by unilateral second premolar agenesis.

Table S3 shows the most common patterns of third molar agenesis in control subjects where agenesis was observed. In the maxilla, as well as in the mandible, bilateral third molar agenesis was the most common pattern (60.5\% and 56.3\%, respectively). In the entire dentition, the most common pattern was the four missing third molars (17.4\%), followed by bilateral third molar agenesis in the mandible, in $14.5 \%$. Table S4 shows the most common patterns of third molar agenesis in the agenesis group, where third molar agenesis was observed. In this group also, bilateral third molar agenesis was the most common pattern within jaws $(74.6 \%$ and $71.0 \%$, in the maxilla and the mandible, respectively). Furthermore, in the entire dentition, the most common pattern was also in this group the four missing third molars $(38.3 \%)$, followed by bilateral third molar agenesis in the mandible (12.3\%).

\section{DISCUSSION}

The purpose of this study was, to explore the patterns of third molar agenesis in a large sample of modern European subjects with and without agenesis of other teeth. The prevalence of third molar agenesis in the agenesis group was $50.8 \%$, which is about 2.5 times higher than in the control group. In the agenesis group, there was a weak correlation of the number of agenesis of other teeth with the number of third molar agenesis within individuals, as well as very weak correlations of third molar agenesis to the number of agenesis of other teeth within quadrants. When considering the percentages of the amount of missing third molars per individual in the control and the agenesis group, there was a tendency towards more missing third molars in the agenesis group. The frequency for bilaterally missing third molars in the agenesis group was about three times higher than the frequency of unilateral absence. The ratio of bilateral to unilateral third molar agenesis was also significantly higher in the agenesis group, compared to the control group.

It could also be useful to note, that based on our findings, in young patients with severe tooth agenesis, the clinician should expect that probably the third molars will also be missing. This should be considered in the treatment planning of severe tooth agenesis cases, which is usually complex and requires a multidisciplinary approach.

Our methodology differs from all previous studies, in terms that we investigated the patterns of third molar agenesis in a large sample with agenesis of other teeth. To our knowledge, all the existing studies divided their groups according to third molar agenesis. Through the latter approach, only a small percentage of the subsequent subsamples had agenesis in teeth other than third molars, and thus, these groups did not have adequate or comparable size to the control groups. Our study tested a large agenesis sample of 303 agenesis individuals, as well as 303

Peer] reviewing PDF | (2020:06:50473:2:0:NEW 15 Oct 2020) 
218

219

220

221

222

223

224

225

226

227

228

229

230

231

232

233

234

235

236

237

238

239

240

241

242

243

244

245

246

247

248

249

250

251

252

253

254

255

256

controls, selected out of a total of around 10000 records. This allowed for findings that are presented for the first time in the literature, such as those related to symmetry or to occurrences within quadrants. Furthermore, the groups were matched for sex and age, accounting for any confounding effects of these factors. For younger individuals, these might be related to the differences in dental maturity between sexes of the same chronological age or to the etiology of tooth absence in older individuals. Furthermore, studies on tooth agenesis (Khalaf et al. 2014), as well as on third molar agenesis (Carter \& Worthington 2015), agree in the higher prevalence of agenesis in females than in males. Differences between sexes were not investigated here, since this was beyond the scope of the present study. A potential effect of the sex factor on the outcomes is not expected because the sample was matched for sex.

The age range that we considered was limited from 12.5 to 40 years old. The minimum limit was defined according to various longitudinal studies that showed the correlation between chronological age and the degree of third molar mineralization using Demirjian's developmental stages. This classification has been widely used and tested to facilitate age estimation. Therefore, the choice of this age limit is considered to be appropriate for our purpose (Caldas et al. 2011; de Oliveira et al. 2012; Karataş et al. 2013; Soares et al. 2015; Zandi et al. 2015). The upper age limit of 40 years was chosen to avoid false positive results due to extraction or tooth loss due to other reasons that could have been registered as agenesis.

We found a prevalence of $50.8 \%$ for third molar agenesis in the agenesis group compared to $20.5 \%$ in the control group. According to a recent meta-analysis (Carter \& Worthington 2015), the worldwide average of third molar agenesis is $22.6 \%$ (21.6\% for Europeans), confirming the validity of our control group. Our results clearly demonstrated that in individuals with agenesis of other teeth, the prevalence of third molar agenesis is higher. This points in the same direction with previous studies that showed an increased prevalence of agenesis of other teeth in individuals with third molar agenesis (Bredy et al. 1991; Celikoglu et al. 2011; Endo et al. 2015).

In our control group, the sequence of the number of missing third molars was similar to that of Carter and Worthington (Carter \& Worthington 2015) that showed the highest prevalence for one missing third molar, followed by two, and four missing third molars. However, the most common amount of missing third molars in the agenesis group was four, followed by two and one third molar. This inconsistency is attributed to the different sample composition. The aforementioned meta-analysis tested third molar agenesis in the general population, meaning that individuals with agenesis of other teeth would be limited. The above findings clearly show that the presence of agenesis, in teeth other than third molars, has a considerable effect on third molar agenesis patterns. Especially, the probability to have four missing third molars increases. This suggests that the third molars might be more vulnerable to genetic factors involved in tooth agenesis, as compared to other tooth types. Indeed, this is also supported by the increased number of missing third molars in the agenesis sample compared to that expected by chance. A recent study analysing data from 172 monozygotic and 112 dizygotic twins concluded that third molar formation is strongly controlled by additive genetic factors, providing further support to 
257 our statements (Trakiniene et al. 2018). This concept is in line with the evolutionary trend in

258

259

260

261

262

263

264

265

266

267

268

269

270

271

272

273

274

275

276

277

278

279

280

281

282

283

284

285

286

287

288

289

290

291

292

293

294

295

humans towards less teeth, and more specifically, less molars (Kavanagh et al. 2007). Facial size has also been reduced during evolution (Bastir et al. 2010). Recent evidence showed that the number of teeth that are formed in a dentition is associated with facial size in modern humans. This indicates that a biological mechanism of tooth number reduction that has evolved during time might still be active and continue to regulate the number of teeth and facial size in a coordinated manner (Oeschger et al. 2020). The findings of the present study, along with the high prevalence of third molar agenesis in the population (Carter \& Worthington 2015) suggest that the third molars might be affected to a higher degree from such mechanisms, compared to other teeth in the dentition.

Furthermore, in terms of developmental timing, the third molar is the last tooth in the molar series and also the last tooth to develop in the dentition. Evidence supports that the last tooth in each tooth series shows more often developmental disturbances, including agenesis, thus being more vulnerable to genetic or environmental factors that might be present during development (Townsend et al. 2009; Gkantidis et al. 2017). This might be another contributing factor relevant to the present results. It has also been shown that overall dental development is delayed in patients with tooth agenesis, compared to controls, with a weak correlation between dental developmental stage and number of missing teeth (Lebbe et al. 2017).

In the agenesis group, the prevalence for bilaterally missing third molars was more than three times higher than in the control group, in the maxilla as well as in the mandible. The ratio of bilateral to unilateral third molar agenesis was significantly higher in the agenesis group compared to the control group. The same was true for all types of symmetry. Furthermore, in the agenesis and the control group, the most common third molar agenesis pattern was four missing third molars, followed by bilateral third molar agenesis in the mandible. This is in line with our previous statement that third molars are more susceptible to genetic or epigenetic factors that cause tooth agenesis, and might more possibly be affected as a whole. The above claim is also supported by the increasing possibility for more missing third molars in the agenesis group than in the controls. Furthermore, very weak correlations were identified between other missing teeth and third molar agenesis within quadrants, suggesting that there are no significant genetic effects limited within quadrants.

A limitation of the study could be that the sample was selected from orthodontic practices, meaning that it may not be representative of the general population. For example, it might be evident that the percentage of severe tooth agenesis occurrences is higher in our sample, since it derived from orthodontic patients, including two university centres. However, the study aimed to test the association of third molar formation with the formation of other teeth using a casecontrol study design. Thus, the study did not aim to represent the general population, but it aimed to test the association of the severity of agenesis of other teeth with third molar formation. Therefore, this is not considered a limitation of the study. On the contrary, it led to adequate number of cases representing the occurrence of severe agenesis $(n=27$ cases with 6 or more

PeerJ reviewing PDF | (2020:06:50473:2:0:NEW 15 Oct 2020) 
296

297

298

299

300

301

302

303

304

305

306

307

308

309

310

311

312

313

314

315

316

317

318

319

320

321

322

323

324

325

326

327

328

329

330

331

332

333

teeth missing, $8.9 \%$ of the agenesis sample). The fact that the sample derived from orthodontic patients, which are thoroughly documented and followed over time, reduces the possibility that certain severe cases might represent non-diagnosed syndromes. Even if limited misdiagnosed cases were present in the sample, our analysis showed very weak correlations of the total number of other missing teeth to the total number of missing third molars. Thus, these cases could have not confounded our findings considerably. Without considering third molars, the present tooth agenesis patterns are comparable to those of other studies presented in the literature (Gkantidis et al. 2017; Khalaf et al. 2014), concerning the most common missing teeth and other tooth agenesis patterns. Regarding other characteristics of an orthodontic population, when considering that malocclusion is endemic in recent years, it is not expected that our sample would highly differ from the general population. Another limitation could be the inclusion of patients up to 40 years old, which might increase the chance to miss information on causes of tooth loss, such as due to extractions. To control for this confounding, according to our inclusion criteria, a case was excluded when the treating doctors judged that the cause for a missing tooth was unclear.

Through this approach, misdiagnosis might not be fully excluded, but it was limited considerably, in order not to critically affect the outcomes. Finally, the present results are based only on subjects of the European population, and thus, they have to be confirmed on other ancestries. However, the study sample originated from places where the white European background is highly represented. Thus, we decided to include only white subjects of European ancestry to avoid confounding.

\section{CONCLUSION}

The present study showed that individuals with non-syndromic tooth agenesis in teeth other than third molars show a higher prevalence of third molar agenesis compared to matched control individuals without agenesis of other teeth. There was also a clear tendency towards more missing third molars in the agenesis group. Furthermore, in the agenesis group, the prevalence for bilaterally missing third molars was more than three times higher than in the control group. The ratio of bilateral to unilateral third molar agenesis was also significantly higher. The above findings indicate that the third molars might be more vulnerable to genetic or epigenetic factors involved in agenesis of other teeth and they are often affected as a whole. These findings seem to be associated with the evolutionary trend in humans towards reduced number of teeth.

\section{REFERENCES}

Abe R, Endo T, and Shimooka S. 2010. Maxillary first molar agenesis and other dental anomalies. Angle Orthod 80:1002-1009. 10.2319/020210-69.1

Banks HV. 1934. Incidence of Third Molar Development. The Angle Orthodontist 4:223-233. 10.1043/0003-3219(1934)004<0223:Iotmd >2.0.Co;2

Bastir M, Rosas A, Stringer C, Cuetara JM, Kruszynski R, Weber GW, Ross CF, and Ravosa MJ. 2010. Effects of brain and facial size on basicranial form in human and primate evolution. J Hum Evol 58:424-431. 10.1016/j.jhevol.2010.03.001 
334

335

336

337

338

339

340

341

342

343

344

345

346

347

348

349

350

351

352

353

354

355

356

357

358

359

360

361

362

363

364

365

366

367

368

369

370

371

372

373

374

375

376

377

Bredy E, Erbring C, and Hubenthal B. 1991. [The incidence of hypodontia with the presence and absence of wisdom teeth]. Dtsch Zahn Mund Kieferheilkd Zentralbl 79:357-363.

Caldas IM, Julio P, Simoes RJ, Matos E, Afonso A, and Magalhaes T. 2011. Chronological age estimation based on third molar development in a Portuguese population. Int J Legal Med 125:235-243. 10.1007/s00414-010-0531-8

Carter K, and Worthington S. 2015. Morphologic and Demographic Predictors of Third Molar Agenesis: A Systematic Review and Meta-analysis. J Dent Res 94:886-894. $10.1177 / 0022034515581644$

Celikoglu M, Bayram M, and Nur M. 2011. Patterns of third-molar agenesis and associated dental anomalies in an orthodontic population. Am J Orthod Dentofacial Orthop 140:856860. 10.1016/j.ajodo.2011.05.021

Celikoglu M, Kazanci F, Miloglu O, Oztek O, Kamak H, and Ceylan I. 2010. Frequency and characteristics of tooth agenesis among an orthodontic patient population. Med Oral Patol Oral Cir Bucal 15:e797-801. 10.4317/medoral.15.e797

de Oliveira FT, Capelozza AL, Lauris JR, and de Bullen IR. 2012. Mineralization of mandibular third molars can estimate chronological age--Brazilian indices. Forensic Sci Int 219:147150. 10.1016/j.forsciint.2011.12.013

Endo S, Sanpei S, Ishida R, Sanpei S, Abe R, and Endo T. 2015. Association between third molar agenesis patterns and agenesis of other teeth in a Japanese orthodontic population. Odontology 103:89-96. 10.1007/s10266-013-0134-1

Endo T, Sanpei S, Komatsuzaki A, Endo S, Takakuwa A, and Oka K. 2013. Patterns of tooth agenesis in Japanese subjects with bilateral agenesis of mandibular second premolars. Odontology 101:216-221. 10.1007/s10266-012-0080-3

Garib DG, Alencar BM, Lauris JR, and Baccetti T. 2010. Agenesis of maxillary lateral incisors and associated dental anomalies. Am J Orthod Dentofacial Orthop 137:732.e731-736; discussion 732-733. 10.1016/j.ajodo.2009.12.024

Garib DG, Peck S, and Gomes SC. 2009. Increased occurrence of dental anomalies associated with second-premolar agenesis. Angle Orthod 79:436-441. 10.2319/021308-87.1

Garn SM, Lewis AB, and Vicinus JH. 1962. Third molar agenesis and reduction in the number of other teeth. J Dent Res 41:717. 10.1177/00220345620410033001

Gkantidis N, Katib H, Oeschger E, Karamolegkou M, Topouzelis N, and Kanavakis G. 2017. Patterns of non-syndromic permanent tooth agenesis in a large orthodontic population. Arch Oral Biol 79:42-47. 10.1016/j.archoralbio.2017.02.020

Karataş OH, Öztürk F, Dedeoğlu N, Çolak C, and Altun O. 2013. Radiographic evaluation of third-molar development in relation to the chronological age of Turkish children in the southwest Eastern Anatolia region. Forensic Sci Int 232:238.e231-235. 10.1016/j.forsciint.2013.07.023

Kavanagh KD, Evans AR, and Jernvall J. 2007. Predicting evolutionary patterns of mammalian teeth from development. Nature 449:427-432. 10.1038/nature06153

Khalaf K, Miskelly J, Voge E, and Macfarlane TV. 2014. Prevalence of hypodontia and associated factors: a systematic review and meta-analysis. J Orthod 41:299-316. 10.1179/1465313314Y.0000000116

Koussoulakou DS, Margaritis LH, and Koussoulakos SL. 2009. A curriculum vitae of teeth: evolution, generation, regeneration. Int J Biol Sci 5:226-243. 10.7150/ijbs.5.226 
Lebbe A, Cadenas de Llano-Pérula M, Thevissen P, Verdonck A, Fieuws S, and Willems G. 2017. Dental development in patients with agenesis. Int J Legal Med 131:537-546. 10.1007/s00414-016-1450-0

Oeschger ES, Kanavakis G, Halazonetis DJ, and Gkantidis N. 2020. Number of teeth is associated with facial size in humans. Sci Rep 10:1820. 10.1038/s41598-020-58565-8

Soares CB, Figueiroa JN, Dantas RM, Kurita LM, Pontual Ados A, Ramos-Perez FM, Perez DE, and Pontual ML. 2015. Evaluation of third molar development in the estimation of chronological age. Forensic Sci Int 254:13-17. 10.1016/j.forsciint.2015.06.022

Townsend G, Harris EF, Lesot H, Clauss F, and Brook A. 2009. Morphogenetic fields within the human dentition: A new: Clinically relevant synthesis of and old concept. Archives of Oral Biology 54: S34-S44.

Trakiniene et al. 2018. Impact of genetics on third molar agenesis. Sci Rep 8:8307. 10.1038/s41598-018-26740-7

van Wijk AJ, and Tan SP. 2006. A numeric code for identifying patterns of human tooth agenesis: a new approach. Eur J Oral Sci 114:97-101. 10.1111/j.1600-0722.2006.00340.x von Elm E, Altman DG, Egger M, Pocock SJ, Gøtzsche PC, and Vandenbroucke JP. 2008. The Strengthening the Reporting of Observational Studies in Epidemiology (STROBE) statement: guidelines for reporting observational studies. J Clin Epidemiol 61:344-349. 10.1016/j.jclinepi.2007.11.008

Zandi M, Shokri A, Malekzadeh H, Amini P, and Shafiey P. 2015. Evaluation of third molar development and its relation to chronological age: a panoramic radiographic study. Oral Maxillofac Surg 19:183-189. 10.1007/s10006-014-0475-0 
Figure 1

Distribution of individuals with different number of missing third molars ( $x$-axis) in the agenesis and the control group.

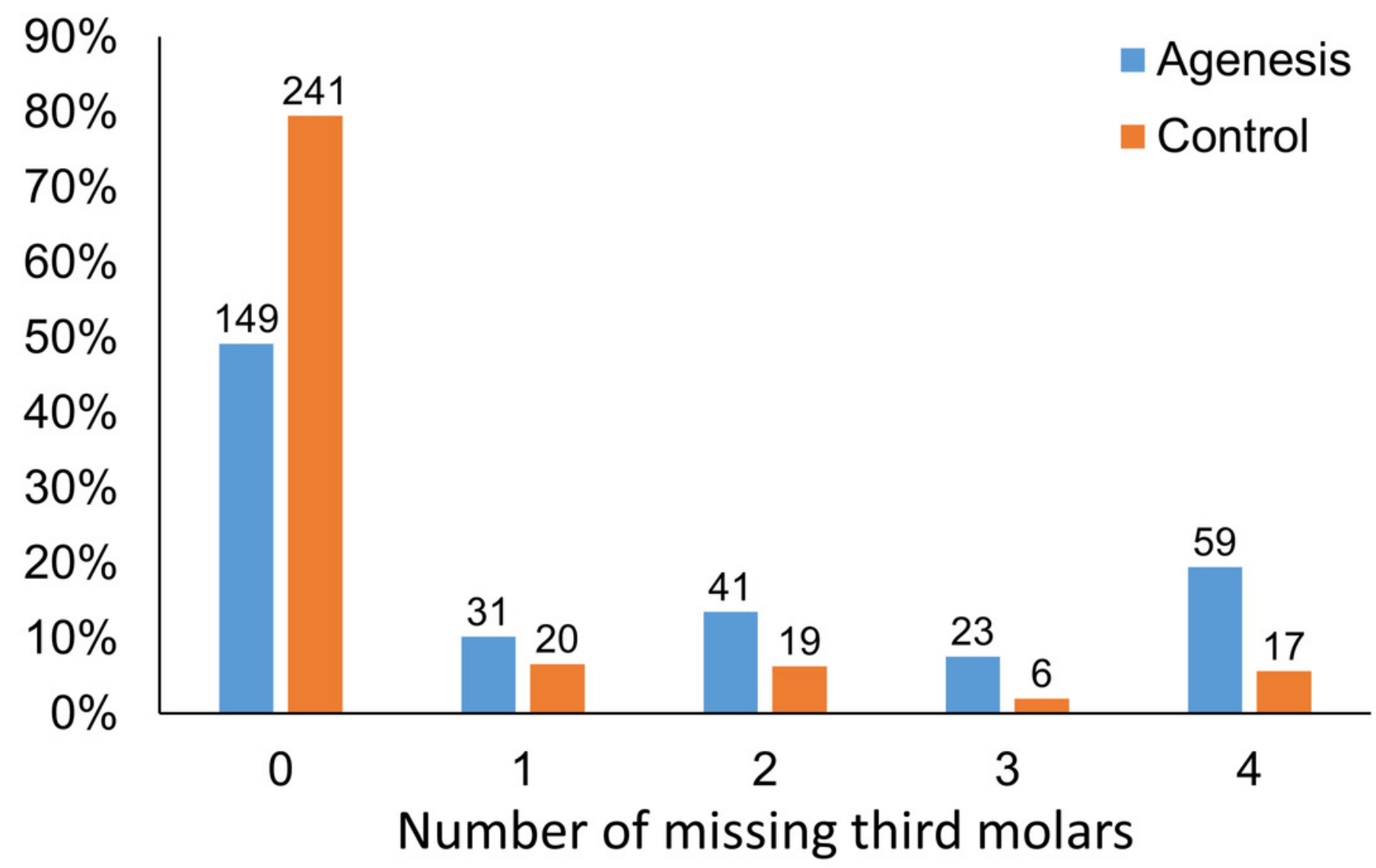




\section{Table 1 (on next page)}

Distribution of missing teeth across quadrant and tooth number. 
1 Table 1 Distribution of missing teeth across quadrant and tooth number.

\begin{tabular}{cccccc}
\hline Tooth number* & $\begin{array}{l}\text { Upper right } \\
(\%)\end{array}$ & $\begin{array}{l}\text { Upper left } \\
(\%)\end{array}$ & $\begin{array}{l}\text { Lower right } \\
(\%)\end{array}$ & $\begin{array}{l}\text { Lower left } \\
(\%)\end{array}$ & Total (\%) \\
\hline Agenesis group & & & & & \\
\hline 1 & $3(0.25)$ & $2(0.2)$ & $29(2.4)$ & $30(2.5)$ & $64(5.3)$ \\
2 & $85(7)$ & $83(6.8)$ & $15(1.2)$ & $18(1.5)$ & $201(16.5)$ \\
3 & $14(1.2)$ & $13(1.1)$ & $5(0.4)$ & $4(0.3)$ & $36(3)$ \\
4 & $20(1.6)$ & $21(1.7)$ & $15(1.2)$ & $15(1.2)$ & $71(5.8)$ \\
5 & $60(4.9)$ & $52(4.3)$ & $117(9.6)$ & $117(9.6)$ & $346(28.4)$ \\
6 & $5(0.4)$ & $4(0.3)$ & $9(0.7)$ & $7(0.6)$ & $25(2.1)$ \\
7 & $13(1.1)$ & $14(1.2)$ & $15(1.2)$ & $14(1.2)$ & $56(4.6)$ \\
8 & $101(8.3)$ & $105(8.6)$ & $104(8.5)$ & $108(8.9)$ & $418(34.3)$ \\
Total & $301(24.7)$ & $294(24.2)$ & $309(25.4)$ & $313(25.7)$ & $1217(100)$ \\
\hline Control group & \multicolumn{5}{l}{} \\
\hline 8 & $37(25.7)$ & $32(22.2)$ & $39(27.1)$ & $36(25)$ & $144(100)$ \\
\hline
\end{tabular}

2 *Tooth number 1 stands for all central incisors $(11,21,31,41)$ and so on. For example, Upper right 1 is 3 tooth 11 in the FDI system. 


\section{Table 2 (on next page)}

Frequency tables that show single tooth agenesis and the prevalence of right sided, left sided or bilateral agenesis in the whole sample $(n=606)$. 
1 Table 2 Frequency tables that show single tooth agenesis and the prevalence of right sided, left

2 sided or bilateral agenesis in the whole sample $(n=606)$.

\begin{tabular}{|c|c|c|c|c|c|}
\hline \multicolumn{6}{|l|}{ Maxilla } \\
\hline Tooth number* & $\begin{array}{l}\text { Present } \\
\text { bilaterally } \\
(\%)\end{array}$ & $\begin{array}{l}\text { Missing right } \\
\text { side (q1) (\%) }\end{array}$ & $\begin{array}{l}\text { Missing left } \\
\text { side }(q 2)(\%)\end{array}$ & $\begin{array}{l}\text { Missing } \\
\text { unilaterally }(\%)\end{array}$ & $\begin{array}{l}\text { Missing } \\
\text { bilaterally (\%) }\end{array}$ \\
\hline \multicolumn{6}{|l|}{ Agenesis group } \\
\hline 1 & $300(99.0)$ & $1(0.3)$ & $0(0.0)$ & $1(0.3)$ & $2(0.7)$ \\
\hline 2 & $194(64.0)$ & $26(8.6)$ & $24(7.9)$ & $50(16.5)$ & $59(19.5)$ \\
\hline 3 & $286(94.4)$ & $4(1.5)$ & $3(1.0)$ & $7(2.5)$ & $10(3.3)$ \\
\hline 4 & $277(91.4)$ & $5(1.7)$ & $6(2.0)$ & $11(3.7)$ & $15(5.0)$ \\
\hline 5 & $234(77.2)$ & $17(5.6)$ & $9(3.0)$ & $26(8.6)$ & $43(14.2)$ \\
\hline 6 & $297(98.0)$ & $2(0.7)$ & $1(0.3)$ & $3(1.0)$ & $3(1.0)$ \\
\hline 7 & $287(94.7)$ & $2(0.7)$ & $3(1.0)$ & $5(1.7)$ & $11(3.6)$ \\
\hline 8 & $185(61.1)$ & $13(4.3)$ & $17(5.6)$ & $30(9.9)$ & $88(29.0)$ \\
\hline \multicolumn{6}{|l|}{ Control group } \\
\hline 8 & $260(85.8)$ & $11(3.6)$ & $6(2.0)$ & $17(5.6)$ & $26(8.6)$ \\
\hline
\end{tabular}

\section{Mandible}

\begin{tabular}{clcccc}
\hline Tooth number & $\begin{array}{l}\text { Present } \\
\text { bilaterally } \\
(\%)\end{array}$ & $\begin{array}{l}\text { Missing right } \\
\text { side }(\mathrm{q} 4)(\%)\end{array}$ & $\begin{array}{l}\text { Missing left } \\
\text { side }(\mathrm{q} 3)(\%)\end{array}$ & $\begin{array}{l}\text { Missing } \\
\text { unilaterally (\%) }\end{array}$ & $\begin{array}{l}\text { Missing } \\
\text { bilaterally }(\%)\end{array}$ \\
\hline Agenesis group & & & & & \\
\hline 1 & $268(88.4)$ & $5(1.7)$ & $6(2.0)$ & $11(3.7)$ & $24(7.9)$ \\
2 & $281(92.7)$ & $4(1.3)$ & $7(2.3)$ & $11(3.6)$ & $11(3.6)$ \\
3 & $297(98.0)$ & $2(0.7)$ & $1(0.3)$ & $3(1.0)$ & $3(1.0)$ \\
4 & $284(93.7)$ & $4(1.3)$ & $4(1.3)$ & $8(2.6)$ & $11(3.6)$ \\
5 & $146(48.2)$ & $40(13.2)$ & $40(13.2)$ & $80(26.4)$ & $77(25.4)$ \\
6 & $292(96.4)$ & $4(1.3)$ & $2(0.7)$ & $6(2.0)$ & $5(1.7)$ \\
7 & $283(93.4)$ & $6(2.0)$ & $5(1.7)$ & $11(3.7)$ & $9(3.0)$ \\
8 & $179(59.1)$ & $16(5.3)$ & $20(6.6)$ & $36(11.9)$ & $88(29.0)$ \\
\hline
\end{tabular}

Control group

$\begin{array}{llllll}8 & 255(84.2) & 12(4.0) & 9(3.0) & 21(7.0) & 27(8.9)\end{array}$

*Tooth number 1 stands for all central incisors $(11,21,31,41)$ and so on. For example, Upper right 1 is tooth 11 in the FDI system. 


\section{Table 3(on next page)}

Most common tooth agenesis patterns in the agenesis group excluding third molars. 
1 Table 3 Most common tooth agenesis patterns in the agenesis group excluding third molars.

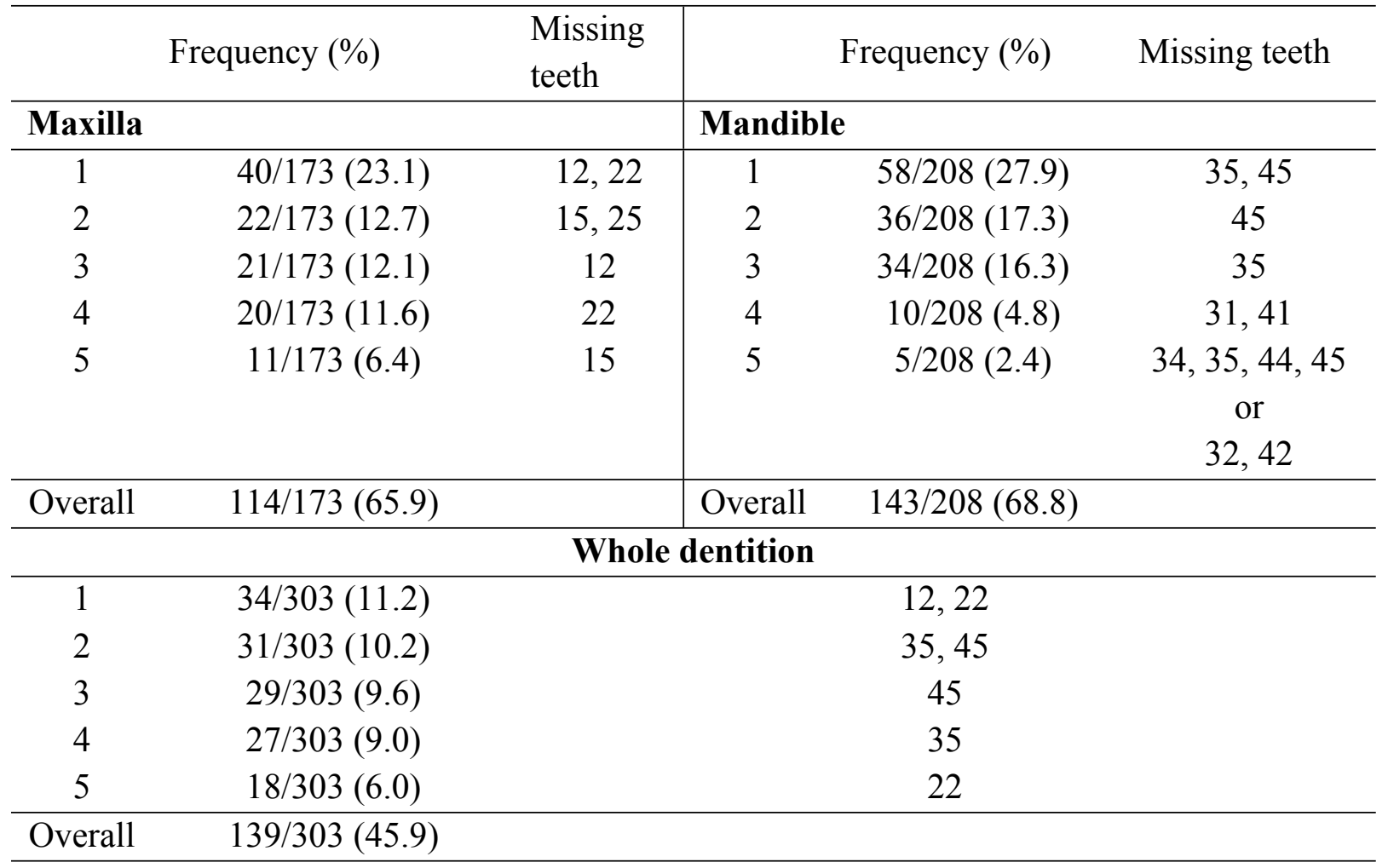




\section{Table 4 (on next page)}

Symmetry of tooth agenesis patterns 
1 Table 4 Symmetry of tooth agenesis patterns.

\begin{tabular}{|c|c|c|c|c|}
\hline & Comparison & & $\begin{array}{l}\text { Symmetry } \\
\text { I }(\%)\end{array}$ & $\begin{array}{l}\text { Symmetry } \\
\text { II }(\%)\end{array}$ \\
\hline \multirow{3}{*}{ Maxilla } & \multirow{3}{*}{ Right vs. left side } & No $3^{\text {rd }}-$ Agenesis & 26.7 & 46.8 \\
\hline & & $3^{\text {rd }}$ - Agenesis & 29.0 & 74.6 \\
\hline & & $3^{\text {rd }}-$ Control & 8.6 & 60.5 \\
\hline \multirow{3}{*}{ Mandible } & \multirow{3}{*}{ Right vs. left side } & No $3^{\text {rd }}-$ Agenesis & 30.0 & 43.8 \\
\hline & & $3^{\text {rd }}-$ Agenesis & 29.0 & 71.0 \\
\hline & & $3^{\text {rd }}-$ Control & 8.9 & 56.3 \\
\hline \multirow{3}{*}{ Left side } & \multirow{3}{*}{ Upper left vs. lower left } & No $3^{\text {rd }}$ - Agenesis & 6.9 & 8.8 \\
\hline & & $3^{\text {rd }}-$ Agenesis & 24.1 & 52.1 \\
\hline & & $3^{\text {rd }}-$ Control & 6.6 & 41.7 \\
\hline \multirow{3}{*}{ Right side } & \multirow{3}{*}{ Upper right vs. lower right } & No $3^{\text {rd }}$ - Agenesis & 7.9 & 9.8 \\
\hline & & $3^{\text {rd }}-$ Agenesis & 24.4 & 56.5 \\
\hline & & $3^{\text {rd }}-$ Control & 8.6 & 52.0 \\
\hline \multirow{3}{*}{ Crossed q1 vs q3 } & \multirow{3}{*}{ Upper right vs. lower left } & No $3^{\text {rd }}$ - Agenesis & 6.9 & 8.71 \\
\hline & & $3^{\text {rd }}-$ Agenesis & 22.1 & 47.2 \\
\hline & & $3^{\text {rd }}-$ Control & 6.6 & 37.7 \\
\hline \multirow{3}{*}{ Crossed q2 vs q4 } & \multirow{3}{*}{ Upper left vs. lower right } & No $3^{\text {rd }}$ - Agenesis & 7.6 & 9.5 \\
\hline & & $3^{\text {rd }}-$ Agenesis & 24.4 & 54.8 \\
\hline & & $3^{\text {rd }}-$ Control & 6.6 & 39.2 \\
\hline
\end{tabular}

2 Symmetry I: percentage relative to the whole sample $(n=303)$ without considering the patterns of no

3 missing teeth as symmetrical. Symmetry II: percentage relative to subsample of subjects with missing

4 teeth in the respective area (i.e. maxilla, mandible etc.). 